\title{
La Evaluación del Desempeño Docente en la Universidad Pedagógica Nacional Francisco Morazán: Una Experiencia en Construcción
}

M.Sc. Bartolomé Chinchilla*

\section{Resumen}

Este artículo presenta una descripción de los procedimientos desarrollados en la evaluación del desempeño docente por la Dirección de Evaluación y Acreditación de la Universidad Pedagógica Nacional Francisco Morazán, como una estrategia de aseguramiento de la mejora de la calidad educativa, producto de ello, se creó el programa de evaluación y mejoramiento de la docencia como una vía fundamental para la atención, estimulación y mejora permanente de las estructuras docentes y académicas de la institución a través de dos ejes fundamentales:

a. La evaluación del desempeño docente.

b. La evaluación de la gestión académica.

La iniciativa de organizar de manera sistemática la evaluación del desempeño docente se inspira en propuestas que vienen cobrando importancia en nuestro país y en el ámbito de la Educación Superior Centroamericana.

En la UPNFM se establecen tres procedimientos para realizar la evaluación docente: Encuesta de opinión al estudiante, Acompañamiento docente y la Autoevaluación del docente.

Palabras Clave: <Desempeño docente $><$ Síntesis valorativa $><$ par acompañante $><$ portafolio docente $><$ plan de mejoramiento de la docencia>.

* Director de Evaluación y Acreditación -UPNFM- 


\section{Antecedentes}

La problemática económica, social y política que actualmente enfrenta la sociedad mundial y, particularmente la latinoamericana, impone retos formidables a las instituciones educativas, especialmente a las universidades. Según el Informe de la UNESCO sobre la Educación Superior en América Latina y el Caribe 20002005 éstos se resumen de la siguiente manera:

- La definición del marco epistemológico desde el cual la universidad latinoamericana y caribeña se piensa a sí misma.

- El esclarecimiento de su papel en la producción y la difusión de saberes.

- La definición de su lugar en la sociedad (social, cultural, político).

- La búsqueda de la equidad y la calidad.

- Las limitaciones de su cultura organizacional y sus formas de funcionamiento.

En ese sentido, es importante recalcar que en la región se están realizando esfuerzos para tratar de responder a las preocupaciones comunes por medio de la conformación de entes especializados en los temas de evaluación y acreditación, así como la realización de foros, congresos, estudios regionales y otro tipo de eventos académicos encaminados a debatir y proponer alternativas de solución a la problemática común.

Una de las variables que se ha identificado como determinante para el logro de mejores niveles de calidad en la gestión universitaria es el "desempeño profesional docente". Por mucho tiempo se había creído que los factores sociales, económicos y culturales ejercían tal influencia para el éxito o fracaso estudiantil, que las instituciones educativas poco podían hacer al respecto, sin embargo esta postura ha cambiado trayendo como consecuencia la implementación de diferentes tipos de programas encaminados a mejorar la labor docente.

Según Valdés Veloz (2000), la evaluación del desempeño de los docentes comenzó a principios de la década de los 30 y desde 
entonces ha sido un área del quehacer educativo especialmente controvertida. Desde fines de la década de los 80 , con el mayor uso de las evaluaciones de desempeño tanto para docentes como para estudiantes, las críticas en la literatura profesional han tendido cada vez más a concentrarse en los aspectos prácticos de las evaluaciones del desempeño.

La evaluación del desempeño docente es un proceso complejo y sensitivo lo que hace que surjan diferentes formas de conceptuarlo. Estas formas se corresponden con el concepto de evaluación que prevalece en la institución educativa en donde el docente labora, los propósitos o fines de la evaluación y los procedimientos que se utilizan para realizarla.

En opinión de Bretel (2002), la evaluación del desempeño docente es un proceso formativo y sumativo a la vez, de construcción de conocimientos a partir de los desempeños docentes reales, con el objetivo de provocar cambios en ellos, desde la consideración axiológica de lo deseable, lo valioso y el deber ser del desempeño docente, de tal suerte que ya no sea vista como una actividad punitiva que censura y desprestigia al docente, sino, por el contrario, aquella que le muestra sus posibilidades y limitaciones en la búsqueda del mejoramiento continuo.

Valdés considera que no sólo son las capacidades pedagógicas del docente las que inciden en el rendimiento del estudiante sino que una combinación de éstas con su emocionalidad, responsabilidad laboral y relaciones interpersonales; de esta manera, se estará evaluando al docente como un ser integral que no solo es portador de conocimientos sino que es capaz de sentir, expresarse y convivir con los demás y que, sus actitudes al respeto inciden también en los estudiantes.

Sin lugar a dudas, una propuesta de evaluación desde esta perspectiva requerirá la superación de los viejos esquemas positivistas pues la comprensión implica "adentrarse" en el objeto de evaluación para, a través de una valoración crítica de sus principales elementos y relaciones internas, obtener conclusiones válidas con respecto al mismo. 
La propuesta de evaluación del desempeño docente de la UPNFM se fundamenta en los planteamientos de los autores citados anteriormente, la que se concreta a través del Programa de Evaluación y Mejoramiento de la Docencia. Dicho programa establece tres procedimientos para realizar la evaluación docente: a) Encuesta de opinión del estudiante, b) Acompañamiento docente y c) la Autoevaluación del docente.

\section{a) Encuesta de opinión del estudiantes}

Según Bretel (1997), los estudiantes se encuentran en una posición privilegiada para proporcionar información acerca de la efectividad de la docencia. Son los únicos que tienen información directa del tipo, naturaleza y calidad de las prácticas docentes que se realizan en el aula.

En el caso de la UPNFM la encuesta de opinión a estudiantes, fue construida con la participación de docentes de ambas Facultades y validada por expertos nacionales e internacionales. La evaluación del desempeño docente se inició a nivel institucional ${ }^{1}$ a finales del 2005, promovida por la Vicerrectoría Académica, coordinada y dirigida por la Dirección de Evaluación y Acreditación con la presentación de la propuesta de un documento marco para realizar dicho proceso. En el 2006 se inicia la consulta a los estudiantes para valorar el desempeño de sus docente con un instrumento impreso, el cual fue revisado y validado un año después por jueces nacionales e internacionales a fin de garantizar su confiabilidad y validez, en el año 2010 el instrumento es revisado por segunda vez y se dio inicio a la evaluación del desempeño docente a través de la modalidad virtual (consulta en línea), solicitándole a los estudiantes que evaluaran al menos a dos de sus profesores. En el mes de julio de 2012 se revisó por tercera vez el instrumento, en esta ocasión participaron docentes y estudiantes representante por carrera. La evaluación realizada en el segundo período académico 2012,

\footnotetext{
1 Anteriormente la evaluación era realizada por los Jefes y Secretarios de Departamento como una acción de Supervisión Docente. En el 2009 con el inicio de autoevaluación de las carreras la DEVA aplicó un instrumento de evaluación a los estudiantes evaluando diferentes factores y entre ellos el desempeño docente.
} 
fue censal es decir que los estudiantes evaluaron a todos sus docentes con quienes recibieron clases en ese período.

\section{b) Acompañamiento docente}

La propuesta de acompañamiento docente se basa en el trabajo colaborativo entre pares. Según Imbernon (2007:150), dicho modelo se caracteriza por el hecho de suscitar la inventiva del docente, así como su capacidad de regularla; la confianza en la capacidad del profesorado de elaborar itinerarios educativos diferenciados, proyectos de innovación y prácticas alternativas. El par acompañante asume un papel de compromiso con la práctica del otro, se involucra en una sociedad de innovación respetando las prácticas educativas en las cuales también puede experimentar y aprender con los otros.

Los componentes del acompañamiento docente van desde la revisión del portafolio del docente, observación de clases, hasta la entrevista con el docente evaluado. La función del par acompañante no es la de juzgar al docente acompañado, sino la de hacer que éste sea auto-crítico, reflexivo y consciente de cómo realiza su actividad docente y en consecuencia ser capaz de mejorar por sí mismo. Es decir, ayudar a crecer profesionalmente al docente.

\section{c) Autoevaluación docente}

La utilización de esta técnica permitiría lograr, en la evaluación del desempeño docente, algunos objetivos importantes como estimular su capacidad de autoanálisis y autocrítica, así como su potencial de autodesarrollo; incrementar su nivel de profesionalidad; promover una cultura innovadora. (Bretel, 2007).

\section{Objetivos}

- Obtener e interpretar información referente al desempeño docente a fin de identificar las fortalezas y oportunidades de mejora. 
- Generar información para orientar el asesoramiento y capacitación con base a las necesidades detectadas.

\section{Metodología}

\section{IV.1 Población}

La población participante está conformada por docentes, jefes de sección, coordinadores de carreras, secretarios y jefes de departamento que laboran en la Universidad Pedagógica Nacional Francisco Morazán y estudiantes del sistema presencial de Tegucigalpa, San Pedro Sula y La Ceiba.

\section{IV.2 Técnicas e instrumentos}

El cuadro 1. Proporciona información sobre las técnicas, las dimensiones de evaluación, estrategia y los instrumentos de evaluación, utilizados para valorar de manera válida y confiable el desempeño profesional del docente.

\section{Cuadro 1}

Relación entre técnicas, dimensiones, estrategias e instrumentos de evaluación utilizados en la evaluación del desempeño docente en la UPNFM

\begin{tabular}{|c|c|c|c|}
\hline TÉCNICAS & $\begin{array}{l}\text { DIMENSIONES DE } \\
\text { EVALUACIÓN }\end{array}$ & ESTRATEGIA & INSTRUMENTO \\
\hline $\begin{array}{l}\text { Observación } \\
\text { de clases }\end{array}$ & $\begin{array}{l}\text { Capacidades } \\
\text { pedagógicas. } \\
\text { Ambiente Pedagógico. } \\
\text { Relaciones } \\
\text { interpersonales. } \\
\text { Responsabilidad } \\
\text { profesional. } \\
\text { Resultados de su labor } \\
\text { educativa. }\end{array}$ & $\begin{array}{l}\text { Acompañamiento } \\
\text { docente (por el jefe, } \\
\text { secretario o equipo } \\
\text { de evaluación de cada } \\
\text { carrera). } \\
\text { Evaluación a través } \\
\text { del par acompañante. }\end{array}$ & $\begin{array}{l}\text { Ficha de } \\
\text { observación de } \\
\text { clases. }\end{array}$ \\
\hline $\begin{array}{l}\text { Encuesta de } \\
\text { opiniones a } \\
\text { estudiantes }\end{array}$ & $\begin{array}{l}\text { Capacidades } \\
\text { pedagógicas } \\
\text { Ambiente Pedagógico } \\
\text { Relaciones } \\
\text { interpersonales } \\
\text { Responsabilidad en el } \\
\text { desempeño de sus } \\
\text { funciones }\end{array}$ & $\begin{array}{l}\text { Consulta de evaluación } \\
\text { en línea }\end{array}$ & $\begin{array}{l}\text { Cuestionario de } \\
\text { evaluación }\end{array}$ \\
\hline
\end{tabular}




\begin{tabular}{|c|c|c|c|}
\hline Portafolio & $\begin{array}{l}\text { Responsabilidad en el } \\
\text { desempeño de sus } \\
\text { funciones }\end{array}$ & $\begin{array}{l}\text { Análisis documental: } \\
\text { Jefe, secretario o } \\
\text { equipo de evaluación } \\
\text { de cada carrera) } \\
\text { Evaluación a través } \\
\text { del par acompañante }\end{array}$ & $\begin{array}{l}\text { Lista de cotejo } \\
\text { Guía de análisis } \\
\text { documental }\end{array}$ \\
\hline $\begin{array}{l}\text { Autoevaluación } \\
\text { del docentes }\end{array}$ & $\begin{array}{l}\text { Capacidades } \\
\text { pedagógicas } \\
\text { Ambiente Pedagógico } \\
\text { Relaciones } \\
\text { interpersonales } \\
\text { Responsabilidad en el } \\
\text { desempeño de sus } \\
\text { funciones } \\
\text { Resultados de su labor } \\
\text { educativa }\end{array}$ & $\begin{array}{l}\text { Preguntas } \\
\text { orientadoras } \\
\text { entregadas al docente } \\
\text { Resultados de las } \\
\text { evaluaciones hechas a } \\
\text { estudiantes }\end{array}$ & $\begin{array}{l}\text { Cuestionario de } \\
\text { evaluación } \\
\text { Informe de } \\
\text { evaluaciones } \\
\text { hechas por } \\
\text { estudiantes }\end{array}$ \\
\hline $\begin{array}{l}\text { Síntesis } \\
\text { valorativa }\end{array}$ & $\begin{array}{l}\text { Capacidades } \\
\text { pedagógicas } \\
\text { Relaciones } \\
\text { interpersonales } \\
\text { Ambiente Pedagógico } \\
\text { Responsabilidad en el } \\
\text { desempeño de sus } \\
\text { funciones } \\
\text { Resultados de su labor } \\
\text { educativa }\end{array}$ & $\begin{array}{l}\text { Entrevista a } \\
\text { docentes, llenado de } \\
\text { síntesis valorativa } \\
\text { Propuesta de plan de } \\
\text { mejoramiento } \\
\text { docente(por carrera e } \\
\text { individual) }\end{array}$ & $\begin{array}{l}\text { Matriz síntesis } \\
\text { valorativa } \\
\text { Formato plan de } \\
\text { mejoramiento }\end{array}$ \\
\hline
\end{tabular}

\section{IV.3 Fuentes de información}

Se utilizan como fuentes de información los estudiantes, los cuales aportan informes valiosos acerca del desempeño del docente en el aula; los jefes de departamento o sección y los coordinadores de carrera quienes hacen una valoración cualitativa del desempeño del docente; las evidencias del portafolio del docente así como, la autoevaluación del docente.

La evaluación del desempeño docente en la UPNFM está concebida como una actividad cíclica en la que cada fin del ciclo se convierte en un nuevo comienzo del proceso. A continuación se describen las etapas del proceso:

- En primer lugar se recaba información sobre el desempeño docente proveniente de diferentes fuentes: estudiantes, jefes de departamento y docentes. 
- Con los insumos de la primera etapa los Jefes de Departamento realizan la Síntesis Valorativa del Desempeño Docente, éste es un resumen de las fortalezas y debilidades detectadas en el desempeño docente a través de las fuentes ya citadas, incluyendo su autoevaluación.

- Utilizando las síntesis valorativas como insumo, los Jefes de Departamentos académicos, elaboran un plan de mejoramiento de la docencia con el fin de fortalecer las áreas débiles y consolidar los aspectos positivos.

- El proceso anterior es revisado de nuevo a la luz de la teoría de la educación y de la evaluación realizando los ajustes que se consideren necesarios.

- Se implementan los planes de mejoramiento registrando las incidencias del proceso.

- Lo anterior permite la consolidación de saberes, tanto teóricos como prácticos, los cuales son sistematizados.

- Los nuevos saberes pueden conducir al diseño e implementación de innovaciones educativas las cuales pueden ser luego compartidas con la comunidad educativa.

- Al realizar una valoración de las innovaciones inicia un nuevo ciclo en la evaluación del desempeño docente.

\section{Principales Hallazgos}

Los resultados generales del desempeño docente son una síntesis de las valoraciones realizadas por los estudiantes a través la consulta en línea ejecutada en el II período académico 2012.

Los datos de registro de la matricula fueron de 7,663 estudiantes, los cuales completaron un total de 17,321 evaluaciones. Evaluando un total de 564 asignaturas en 1,083 Secciones, que corresponden a 406 profesores evaluados. 


\section{Cuadro 2}

Resumen del número de docentes evaluados en las distintas asignaturas por los estudiantes del sistema presencial, en el II período académico de 2012

\begin{tabular}{|l|cccc|}
\hline Sede & Matrícula & $\begin{array}{c}\mathrm{N}^{\circ} \text { de } \\
\text { Evaluaciones }\end{array}$ & $\begin{array}{c}\text { Asignaturas } \\
\text { evaluadas }\end{array}$ & $\begin{array}{c}\mathrm{N}^{\circ} \text { de } \\
\text { profesores } \\
\text { evaluados }\end{array}$ \\
\hline Tegucigalpa & 4,928 & 11,125 & 719 & 278 \\
\hline San Pedro Sula & 2,305 & 4,725 & 319 & 108 \\
\hline La Ceiba & 430 & 1,466 & 45 & 20 \\
\hline
\end{tabular}

Para efectos del análisis, se respeta la estructura del cuestionario el cual considera 6 aspectos básicos en la evaluación del desempeño docente: Capacidades pedagógicas, Ambiente Pedagógico, Responsabilidad Profesional, Relaciones interpersonales, Resultados de la labor educativa, y La Autoevaluación del estudiante (este apartado no afecta el promedio de la evaluación obtenida por el docente).

En general las preguntas que integran el cuestionario están referidas a la frecuencia o al nivel de satisfacción, con ítems en una escala de respuesta tipo Likert de 4 puntos considerando la escala de 1 a 4 y NA; donde 1 representa el valor más bajo o negativo, 4 la valoración más alta o positiva y NA cuando la pregunta no aplica. Presentando los promedios obtenidos en cada uno de los ítems del cuestionario y un promedio general del desempeño por dimensión evaluada.

Se considera un rendimiento excelente, cuando el promedio obtenido oscila entre 3.88 - 4.00 (97-100\%); destacado cuando el promedio oscila entre 3.64 - 3.87 (91-96.75\%); aceptable cuando el promedio oscila entre 3.00 - 3.63 (75-90.75\%); y necesita mejorar si el promedio es igual o menor que 2.99 ( $\leq 74.75 \%)$.

Con los datos registrados en la gráfica 1 , se puede inferir que los estudiantes en las diferentes sedes valoraron a sus docentes de forma aceptable, en la mayoría de las dimensiones evaluadas (capacidades pedagógicas, ambiente pedagógico, responsabilidad profesional, resultados de la labor educativa) y de forma destacada 
la dimensión sobre las relaciones interpersonales, excepto en la sede de La Ceiba la cual recibió una valoración aceptable (3.60).

Los estudiantes se autoevaluaron obteniendo una valoración destacada, con un promedio de 3.78 en Tegucigalpa y San Pedro Sula, y 3.87 en La Ceiba.

\section{Gráfica 1}

Resultados generales de la evaluación hecha por los estudiantes a los docentes en las diferentes sedes del sistema presencial de la UPNFM

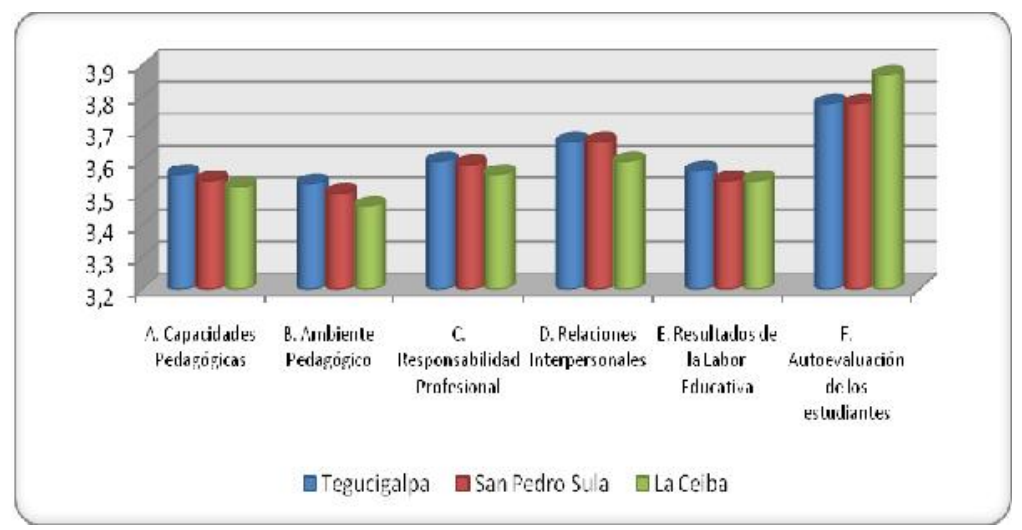

\section{Conclusiones}

- Al considerar los promedios de evaluación general por sede se identifica que estas se ubican en la categoría aceptable, sin embargo es importante aclarar que estos promedios corresponden al límite mayor de esta categoría. Al hacer la conversión a una nota porcentual se aprecia que la sede de Tegucigalpa fue la mayor valorada (89.50\%), San Pedro Sula con $89.15 \%$ y La Ceiba con $88.40 \%$. Al comparar estos datos con el año anterior (2011) se observa una mejora significativa, por lo que se puede inferir que las jornadas de socialización de los resultados, la elaboración de la síntesis valorativa y los planes de mejoramiento de la docencia están incidiendo positivamente en la mejora del desempeño docente.

- El socializar con los docentes el proceso de evaluación del desempeño docente y el involucrarlos en la elaboración, revisión 
y validación de los instrumentos de evaluación han sido determinantes para que este proceso sea aceptado por los docentes y no sea visto como un proceso impuesto por las autoridades, sino como una necesidad para el aseguramiento de la calidad educativa y la mejora personal.

- El generar la síntesis valorativa por carrera, ha permitido realizar una valoración más completa del desempeño docente, ya que es una herramienta útil para sistematizar y triangular los resultados de la evaluación y para la elaboración del plan de mejoramiento de la docencia.

- El establecer un mecanismo de monitoreo a la propuesta de los planes de mejoramiento de la docencia garantiza en gran medida la ejecución de las acciones plasmada en dicho plan. 


\section{Referencias}

- BRETEL, L. “Consideraciones y propuestas para el diseño de un sistema de evaluación del desempeño docente en el marco de una redefinición de la carrera Magisterial". Enero-2002. En http: / / espanol.geocities.com / cne_magisterio/3 /1.1.e_Luis Bretel. htm.

- IMBERNON, F. “Asesorar o Dirigir. El papel del Asesor/A Colaborativo en una Formación Permanente Centrada en el Profesorado y en el Contexto". En ttp://dialnet.unirioja.es / servlet / articulo? código $=2212328$

- RODRÍGUEZ, C. Abel. Los maestros, protagonistas del cambio. Convenio Andrés Bello. Seminario de Integración Educativa Andrés Bello. Noviembre, 1999.

- VALDÉS V., HÉCTOR. “En un mundo de cambios rápidos, sólo el fomento de la innovación en las escuelas permitirá al sistema educacional mantenerse al día con los otros sectores". Ponencia presentada en el Encuentro Iberoamericano sobre Evaluación del Desempeño Docente. OEI, México, 23 al 25 de mayo de 2000.

- DEVA-UPNFM, Programa de Evaluación y Mejoramiento de la Docencia, 2005.

- SUAZO, A; CHINCHILLA, B. Y RODRÍGUEZ, D. Modelo de Evaluación del Desempeño Docente: Caso de la UPNFM. EN PRECONGRESO UPNFM - CSUCA, Tegucigalpa Honduras, 2010. 